\title{
Acessibilidade no turismo e hotelaria: desafios contemporâneos
}

\section{Jenny Sousa ${ }^{a}$, Catarina Mangas ${ }^{\mathrm{b}}$}

aESECS, CICS.NOVA.IPLeiria-iACT, CI\&DEI, Politécnico de Leiria, Portugal, jenny.sousa@ipleiria.pt, ${ }^{b} E S E C S$, CICS.NOVA.IPLeiria-iACT, CI\&DEI, Politécnico de Leiria, Portugal, catarina.mangas@ipleiria.pt

\begin{abstract}
Resumo
Na sociedade contemporânea, o turismo detém uma ligação muito estreita com a realização pessoal e o bem-estar dos indivíduos, convertendo-se numa prática social e cultural, que movimenta milhões de pessoas em todo o mundo.
\end{abstract}

No entanto, e apesar da crescente democratização, este fenómeno ainda não é para Todos. São muitos os cidadãos que pelas suas caraterísticas e incapacidades se veem impossibilitados de usufruir, de forma equitativa, dos espaços e dos equipamentos turísticos, designadamente hoteleiros. Neste exercício de massificação do turismo, as pessoas com deficiência surgem enquanto segmento do mercado ainda pouco contemplado, mas cada vez mais emergente e exigente.

Com efeito, as pessoas com deficiência procuram um nível de autonomia cada vez maior e, neste quadro, as unidades hoteleiras veem-se perante um novo paradigma que as desafia, dentro daquilo que é também a rentabilidade financeira e o aumento de valor, a criar ambientes integradores e seguros que possam ser utilizados por todas as pessoas, na sua maior extensão possivel.

Falamos, pois, da criação de ambientes que respeitem os princípios do Desenho Universal, que no contexto da hotelaria diz respeito a muito mais do que a simples adaptação de espaços físicos para pessoas com deficiência, mas antes ao planeamento de todos os aspetos da vida do hotel.

Face ao exposto, com este trabalho, pretendemos realizar uma reflexão teórica que, cruzada com uma análise de natureza mais prática, estimule o debate e aumente o conhecimento sobre a importância do Desenho Universal no âmbito de uma hotelaria realmente para Todos, numa perspetiva de oportunidade de rentabilização e vantagem competitiva.

Palavras-chave: Turismo acessivel, Hotelaria, Desenho Universal, Bemestar pessoal e social. 


\section{Introdução}

As sociedades ocidentais, e as que são diretamente influenciadas por elas, viveram nas últimas décadas profundas transformações culturais, que influenciaram novos estilos de vida e novas formas de percecionar e ocupar o tempo livre. Com efeito, o tempo livre, enquanto tempo disponível que resta depois de cumpridas as obrigações laborais, ultrapassa hoje o período de descanso. Na verdade, o tempo é cada vez mais percebido como um território fértil para a vivência de experiências de lazer e de ócio, enquanto espaço privilegiado de desenvolvimento de capacidades e potencialidades, que permitem às pessoas sentirem-se bem, ativas e incluídas socialmente (Sousa, 2019). Assim, e embora as práticas de lazer e de ócio sejam experiências privadas e subjetivas, a verdade é que se consubstânciam na relação com os outros e com os espaços, constituindo-se estes últimos em importantes meios de realização (Lázaro, Doistua \& Romero, 2018). Neste sentido, para o indivíduo contemporâneo, o tempo de lazer/ócio é um espaço de infinitas possibilidades de desenvolvimento pessoal e social, constituindo-se, cada vez mais, enquanto componente vital de bem-estar pessoal e social.

Neste quadro, o turismo surge enquanto elemento preponderante naquilo que são as práticas de lazer e de ócio. Falamos do turismo enquanto experiência social e cultural, que se concretiza através da relação com os lugares e com as pessoas.

Temos consciência de que o turismo é um conceito complexo e que pode ser perspetivado de variadas formas, dependendo do escopo que estiver a ser utilizado. Com efeito, podemos classificá-lo como tendo diversos tipos: turismo cultural, turismo de natureza, turismo religioso, turismo rural, turismo de mar, que na verdade se refere às diversas estruturas que organizam a oferta e o correspondente produto diferenciador. Todavia, também podemos estudá-lo numa perspetiva de economia do turismo, salientando o capital turístico em relação ao património e à geração de valor (Queirós, 2017). Podemos, ainda, encará-lo desde uma perspetiva de turismo responsável e sustentável, onde a inclusão social tem um papel importante, conforme explica a Organização Mundial do Turismo, no trabalho Global Report on Inclusive Tourism Destinations - Model and Success Stories (World Tourism Organization, 2018).

Perante toda esta complexidade, recorremos às palavras de Margarita Barretto para nos ajudar a situar o turismo naquela que é a principal perspetiva que lhe é atribuída neste trabalho: "turismo é movimento de pessoas, é um fenómeno que envolve, antes de mais nada, gente" (2014,p.7). Gente que viaja e gente que recebe. Na realidade, parece ser ponto assente que o turismo tem um profundo impacto no bem-estar do indivíduo contemporâneo e que apresenta um grande potencial para quebrar barreiras e favorecer a inclusão social (Coelho \& Guimarães, 2012). Para além disso, o turismo é um poderoso fator de 
desenvolvimento, numa ótica de processo globalizador. Neste movimento, e principalmente com a expansão do turismo de massas, as pessoas com deficiência passaram a ter uma voz ativa. Assim, os vários agentes do turismo passaram a olhar para a questão da acessibilidade no âmbito do reforço da competitividade dos destinos e dos locais, numa perspetiva de turismo para Todos. Nesta linha de pensamento, emergem novos desafios, designadamente para os estabelecimentos de alojamento, ao identificarem um potencial cada vez maior naquele que foi durante muito tempo considerado um nicho de mercado, ou seja, as pessoas com deficiência.

Na verdade, a taxa de deficiência ou de incapacidade entre as pessoas que viajam tem vindo a aumentar nos últimos anos (Turismo de Portugal, 2017), refletindo-se, por isso, num aumento da procura de ambientes acessíveis, desde as infraestruturas aos serviços, aumentando o valor do turismo acessível no mercado do turismo. Neste quadro, emerge o Desenho Universal, enquanto meio de criar produtos, serviços e ambientes confortáveis, sustentáveis e seguros para Todos (Beltramini \& Júnior, 2018) porque permite uma utilização equitativa, simples e intuitiva.

Assim sendo, procurou-se, neste trabalho, perceber que reflexos o novo paradigma detém no contexto português. Para tal, foi realizada uma análise crítica baseada em documentos de referência cruzada com uma pesquisa online em torno das práticas que estão a ser desenvolvidas por organismos de referência, unidades hoteleiras, bem como, por associações que desenvolvem ações na área da deficiência e incapacidade. Neste sentido, começamos por discutir o turismo acessível enquanto paradigma contemporâneo de turismo para Todos, abordando, num primeiro momento, o conceito de turismo acessível, e especificando, num segundo momento, a reflexão acerca da importância do Desenho Universal nesta equação de turismo acessível e inclusivo, trazendo à discussão os exemplos encontrados na pesquisa realizada.

\section{Um novo público e um novo paradigma: o turismo acessível como turismo para Todos}

\subsection{Turismo acessível: novas relações, novos sentidos de cidadania}

É oportuno relembrar que hoje, mais do que nunca, o turismo é um bem social cujos efeitos, desde o ponto de vista pessoal e social, são evidentes (Queirós, 2017; Turismo de Portugal, 2013). Assim, a prática do turismo deve estar ao alcance de qualquer cidadão, sem exclusão de qualquer tipo de grupo da população. Este aspeto encontra eco na própria legislação portuguesa, Lei no 38/2004, de 18 de Agosto, no artigo 38 , que prevê o direiro das pessoas com deficiência à fruição dos tempos livres mediante, nomeadamente, a criação 
de estruturas adequadas e formas de apoio social. Embora exista uma consciência generalizada deste facto, a realidade demonstra que as pessoas com deficiência se deparam com maiores dificuldades em escolher livremente as atividades de lazer/ócio que querem realizar.

Quando falamos de pessoa com deficiência, referimos-nos a um conjunto de tipologias no âmbito das deficiências existentes, tais como: deficiência visual; deficiência auditiva; deficiência intelectual; deficiência múltipla; deficiência motora e mobilidade reduzida, que, por sua vez se subdividem em categorias, atendendo às especificidades de cada tipo de deficiência (Direção de Estudos e Planeamento Estratégico, 2012). Portanto, falar de pessoa com deficiência "significa qualquer pessoa cuja participação plena e efetiva, em pé de igualdade com os outros, em viagens, alojamento e outros serviços turísticos é dificultada pelas barreiras existentes no espaço físico que se encontram e pelas barreiras da mentalidade" (Turismo de Portugal, 2013, s.p).

Estamos, destarte, a referir-nos a quem tem diversidade funcional (Organização Mundial de Saúde, 2011) e necessita de lugares adaptados e acessíveis. Falamos, pois, de turismo acessível, ou seja, não "apenas à criação da acessibilidade das pessoas com deficiência/incapacidade, mas também à criação de ambientes de desenho universal que possam apoiar as pessoas com alguma incapacidade temporária [...] bem como a criação de um ambiente mais seguro para a população trabalhadora" (Turismo de Portugal, 2013, s.p).

Em rigor, o turismo acessível surge assente num quadro de referências promotoras de um pensamento e de uma ação ajustadas a Todos, onde o Desenho Universal tem principal destaque. Trata-se, então, de uma nova forma de pensar o turismo, que decorre de um processo evolutivo que alia captação de turistas e preocupações societais.

Em linha com o exposto, percebe-se que, por um lado, este novo paradigma impõe novos desafios aos agentes ligados ao turismo - nomeadamente aos estabelecimentos de alojamento, porque implica reestruturação - mas que, por outro, o próprio mercado percebe, cada vez mais, o potencial de pensar o turismo tendo em conta as necessidades de cada um. Por isso, a noção de Desenho Universal tem acolhido cada vez mais o interesse dos políticos, dos empresários e dos clientes em geral.

\subsection{O Desenho Universal no âmbito do turismo e dos estabelecimentos de alojamento}

Falar de turismo acessível implica abordar também os estabelecimentos de alojamento. $\mathrm{Na}$ verdade, a hospedagem é um elemento quase inerente à viagem e os hotéis acabam por ser um fator crucial nesta equação de usufruto dos tempos livres. Se um hotel estiver apto a receber qualquer cidadão, quer tenha ou não limitações, é um local para Todos e isso converte-se num atrativo de negócio. 
Como a procura do turismo acessível é crescente, este deve ser visto como uma oportunidade e não uma obrigação, tal como alerta o Turismo de Portugal (2013). A respeito deste assunto, o mesmo organismo explica também que se "a indústria do turismo quer manter e desenvolver a sua qualidade, sustentabilidade e competitividade, deve apoiar e desenvolver o turismo acessível", beneficiando-se, desta forma, todos os envolvidos porque "à medida que mais pessoas têm a oportunidade de viajar, a indústria do turismo recebe mais visitantes, as estadias são mais longas e os gastos aumentam" (Turismo de Portugal, 2013, p.4). Por isso mesmo é que esta questão não pode ser exclusiva das autoridades públicas, mas também apanágio de todos os agentes económicos do setor.

Tendo esta ideia como pano de fundo, o conceito de Desenho Universal ganha cada vez mais destaque nas agendas políticas e nas práticas diárias nos vários setores do turismo. Isto acontece porque o Desenho Universal é um processo de tal forma completo e adaptado às várias realidades "que aumenta a segurança, funcionalidade, saúde e participação social, através do design e a operação de ambientes, produtos e sistemas em resposta à diversidade de pessoas e habilidades" (Organização Mundial de Saúde, 2011, p. 178). No entanto, e conforme se explica no mesmo documento, "a funcionalidade [...] não é o único objetivo do desenho universal, e "adaptação e design especializado" são uma parte do fornecimento personalizado e escolha, que pode ser essencial para lidar com a diversidade. Outros termos coincidentes para o mesmo conceito geral são "design para Todos" e "design inclusivo"” (Organização Mundial de Saúde, 2011, p. 178).

Se considerarmos, mais especificamente, os equipamentos hoteleiros, o Desenho Universal permite aumentar a autonomia, o que se vai refletir num maior grau de independência e de satisfação dos indivíduos. Se todos os clientes puderem utilizar as diversas áreas da unidade hoteleira, desde o restaurante, ao bar, piscina, sala de reuniões, entre outros, mais eficaz e rentável será a sua gestão (Turismo de Portugal, 2012). Afinal, se um hotel tem o propósito de receber qualquer pessoa, tem de a atender com conforto, respeito, dignidade e praticidade, parâmetros que vão ao encontro do conceito do Desenho Universal (Beltramini \& Junior, 2018).

O Desenho Universal assenta em sete princípios que garantem ambientes e produtos especiais não só para pessoas com deficiência, mas para Todos, assegurando que qualquer indivíduo possa utilizar com segurança e autonomia os diversos espaços e objetos: o uso equitativo (Igualitário), o uso flexível (Adaptável), o uso simples e intuitivo (Óbvio), informação de fácil percepção (Conhecido), tolerância ao erro (Seguro), esforço físico mínimo (Sem esforço) e dimensionamento de espaços para acesso e uso abrangente (Abrangente) (Carletto \& Cambiaghi, 2015).

Esta vantagem competitiva que o Desenho Universal oferece ao setor do turismo, e às unidades hoteleiras, está bem presente no contexto português, onde se tem verificado uma 
evolução nos últimos anos, coadunando-se com uma mudança de pensamento e de paradigma. Conforme se anuncia na página web do Turismo de Portugal (2020), no texto enquadrador do Programa All for All - Portuguese Tourism, «a aposta na acessibilidade está diretamente associada ao propósito do país: "Receber Bem"».

Nessa mesma página é explicado que este Programa, All for All - Portuguese Tourism, da iniciativa do Turismo de Portugal, "procura mobilizar a indústria do turismo para, numa atuação concertada, tornar Portugal um destino turístico para todos" e mostra como o turismo português está em mudança. Outras iniciativas nasceram nos últimos anos, no desiderato de dar resposta aos diversos desafios colocados às pessoas com deficiência que querem viajar e fazer turismo em Portugal.

Um dos exemplos é a Accessible Portugal que tem vindo a ser desenvolvida com o intuito de promover o Turismo Acessível para Todos, em território nacional. Apresenta-se como uma entidade ao serviço da qualificação da oferta e da procura turísticas, sem preterir nenhum segmento do mercado em função das suas características específicas. Para além disso, acaba por ter um papel de relevo no envolvimento e na formação dos agentes turísticos e na sensibilização das comunidades em geral. Um dos projetos promovidos pela Accessible Portugal é o projeto AccessTUR - Centro de Portugal. Trata-se de um projeto de promoção do turismo acessível e inclusão social, promovido com o apoio da Turismo do Centro e das oito Comunidades Intermunicipais (CIM's) que compõem o território. Para além deste serviço, apresenta também o projeto Turn4All.

O Tur4All é uma plataforma - website e app - de divulgação da oferta turística acessível em Portugal. Tem como principal objetivo melhorar a experiência turística das pessoas com necessidades especiais e como missão operacionalizar, de forma objetiva e atualizada, as condições reais de acessiblidade da oferta turística.

Para além destas iniciativas, pensadas a partir de entidades públicas, também as próprias associações de pessoas com deficiência têm vindo a deter um papel cada vez mais preponderante neste paradigma do Turismo Acessível. Um exemplo é o site Portugal Acessível da Associação Salvador, que pretende ser um guia nacional de referência na disponibilização de informação sobre a acessibilidade física em diferentes tipos de espaços em Portugal, onde o alojamento tem um papel importante.

Assim, e tal como foi sendo frisado ao longo deste trabalho, de entre os vários agentes que compõem a cadeia turística, as unidades hoteleiras destacam-se enquanto elementos cruciais no turismo acessível e também elas revelam uma preocupação crescente com o Desenho Universal. Exemplos como o Real Abadia Congress \& Spa Hotel; Essence inn Marianus ou os hotéis NATIVE ilustram um conjunto cada vez maior de equipamentos e espaços que procuram responder às diversas necessidades dos seus clientes. 
Desta forma, e em jeito de síntese, no que se refere ao setor do turismo português denota-se uma maior mobilização junto dos agentes das diversas áreas, evidenciando-se, cada vez mais, uma atuação concertada rumo à acessibilidade da oferta turística nacional.

\section{Conclusões}

Sabemos que a ocupação saudável dos tempos livres é fundamental na promoção da saúde e do bem-estar do ser humano. Neste quadro, o turismo emerge enquanto atividade privilegiada de acesso ao lazer/ócio, que apenas se dá na sua totalidade se existirem estratégias, ações e recursos criados para eliminar barreiras, sejam elas físicas, intelectuais ou sociais, para permitir o usufruto de todas pessoas, independentemente das suas limitações. Sendo o turismo uma atividade económica que depende de uma infinidade de serviços especializados, muitas vezes o que diferencia um produto é a capacidade de satisfazer as necessidades do cliente. Este é o pano de fundo do turismo acessível, que se preocupa em responder às expetativas de Todos, para que Todos - quer tenham, ou não, necessidades especiais - para que possam exercer o seu direito de desfrutar do lazer. Neste contexto, pensar o setor do turismo, designadamente as unidades hoteleiras, sob a égide do Desenho Universal, é garantir que Todos os cidadãos podem ser recebidos, podendo aumentar significativamente a procura. Em contexto português, e tal como se verificou na pesquisa desenvolvida, esta realidade é cada vez mais visível. Diversos organismos associados ao turismo e a outros agentes económicos do setor assumem de uma forma cada vez mais preponderante a missão de operacionalizar a acessibilidade dos espaços e dos serviços, permitindo aumentar a qualidade das experiências vividas pelos turistas e pelas comunidades locais, beneficiando Todos.

\section{Referências}

Accessible Portugal (2019). Turismo Acessivel. Disponível em http://accessibleportugal.com/.

Associação Salvador (2020). Portugal Acessivel. Disponível em http://www.portugalacessivel.pt/default/module/id/117.

Barretto, M. (2014). Manual de iniciação ao estudo do turismo. Campinas: Papirus Editora.

Beltramini, L. \& Júnior, A. (2018). Aplicação do desenho universal: uma análise da acessibilidade em hotéis de João Pessoa. In Atas do Congresso Luso-Brasileiro para o Planeamento Urbano, Regional, Integrado e Sustentável. Coimbra - Portugal, 24, 25 e 26 de outubro de 2018 (pp.1-13).

Carletto, A. \& Cambiaghi, S. (2015). Desenho Universal- Um conceito para todos. Disponível em https://www.maragabrilli.com.br/wp-content/uploads/2016/01/universal_web-1.pdf 
Coelho, M. \& Guimarães, M. (2012). Turismo Inclusivo e Lazer em Espaços Públicos. In Atas do IX Seminário da Associação Nacional Pesquisa e Pós-Graduação em Turismo. São Paulo: Universidade Anhembi Morumbi, 30 de agosto e 01 de setembro de 2012 (pp.1-12).

Direção de Estudos e Planeamento Estratégico (2012). Guia de Boas Práticas de Acessibilidade na Hotelaria. Lisboa: Turismo de Portugal. Retrieved from https://travelbi.turismodeportugal.pt/ptpt/Documents/Sustentabilidade/Boas $\% 20 \mathrm{Pr} \% \mathrm{C} 3 \%$ A1ticas/guia-boas-praticas-acessibilidadehotelaria.pdf

Essence inn Marianos (2020). Disponível em https://www.emarianos.com/hotel.

Lázaro, Y., Doistua, J. \& Romero, S. (2018). El ócio a lo largo de la vida: un elemento de cohesión social. In A. Ortuzar \& A. Elizondo (Ed.), Ocio y participación social en entornos comunitários (pp. 61-75). Logroño: Universidad de la Rioja.

Lei $n^{\circ}$ 38/2004, de 18 de Agosto. Diário da República n. ${ }^{\circ}$ 194/2004, Série I-A de 2004-08-18 Retrieved from https://dre.pt/pesquisa/-/search/480708/details/maximized

Native (2020). Disponível em https://www.nativehotels.org/pt-pt/.

Organização Mundial de Saúde (2011). Relatório Mundial sobre a Deficiência. São Paulo: Governo do Estado de São Paulo - Secretaria dos Direitos da Pessoa com Deficiência. Disponível em https://apps.who.int/iris/bitstream/handle/10665/44575/9788564047020 por.pdf? sequence=4\&is Allowed $=\mathrm{y}$

Queirós, A. (2017). Turismo, na mudança de paradigmas. In M.A. Lousada \& V. Ambrósio (Eds.), Literatura, viagens e turismo cultural no Brasil, em França e em Portugal. Lisboa: Centro de Estudos Geográficos, Instituto de Geografia e Ordenamento do Território, Universidade de Lisboa.

Real Abadia Congress \& Spa Hotel (2020). Disponível em https://www.realabadiahotel.pt/pt/turismoacessivel.

Sousa, J. (2019). Participação Sociocultural, Ócio, Acessibilidade e Envelhecimento Ativo no contexto de Idosos Institucionalizados. Revista Subjetividades, 19 (2), pp. 1-14. ISSN: 2359-0769. DOI: 10.5020/23590777.rs.v19i2.e9237.

Turismo de Portugal (2013). Turismo Acessivel para Todos - Recomendações da OMT. Disponível em http://business.turismodeportugal.pt/SiteCollectionDocuments/all-for-all/recomendacoesomt-turismo-acessivel-para-todos.pdf

Turismo de Portugal (2017). Estratégia Turismo 2027 - Liderar o Turismo do Futuro. Disponível em http://institucional.turismodeportugal.pt/SiteCollectionDocuments/estrategia/estrategia-turismo2027.pdf

Turismo de Portugal (2020). Programa All for All - Portuguese Tourism. Disponível em http://business.turismodeportugal.pt/pt/Conhecer/estrategia-turismo/programasiniciativas/Paginas/all-for-all-portuguese-tourism.aspx

Tur4All (2020). TUR4all - Plataforma de recursos turísticos acessiveis para todos. Disponível em https://www.tur4all.pt/. 
World Tourism Organization (2018). Global Report on Inclusive Tourism Destinations - Model and Success Stories. Madrid: UNWTO. DOI: https://doi.org/10.18111/9789284420094. 